\title{
Gastrectomía en manga robótica y laparoscópica: Resultados tempranos de un estudio en población mexicana
}

\author{
Robotic and laparoscopic sleeve gastrectomy: Early \\ results of a study in a Mexican population
}

\author{
Omar Felipe Gaytán Fuentes, ${ }^{\star}$ Israel Abraham Gaytán Fuentes, ${ }^{\ddagger}$ Gustavo Andrés Ayala Ventura,, Edith Barajas Galicia, ${ }^{\llbracket}$ \\ José Guerrero Cantera," Adriana Itzel Almazán Hernández," Omar Ricardo Quiroz Rodríguez" \\ * Médico adscrito al Servicio de Cirugía General. Centro Médico Nacional 20 de Noviembre, ISSSTE. Clínica OBEDI. Ciudad de México. \\ ¥ Investigador Clínico Posdoctoral. Clínica OBEDI. Ciudad de México. \\ $\S$ Coordinación General de Docencia. Hospital Carlos Andrade Marín. Quito, Ecuador. \\ "Médico adscrito al Servicio de Cirugía General. Hospital Central Norte de PEMEX. Ciudad de México. \\ " Investigador Clínico OBEDI. Ciudad de México.
}

RESUMEN

Introducción: La gastrectomía en manga ha ganado popularidad durante la última década debido a su seguridad, viabilidad y buenos resultados. El propósito de este estudio es describir nuestros resultados a corto plazo con estos procedimientos. Material y métodos: Este estudio es una revisión retrospectiva no aleatorizada de 36 pacientes que se sometieron a una gastrectomía en manga de mínimo acceso en el Centro Médico Nacional 20 de Noviembre de enero de 2015 a diciembre de 2018. Un total de 18 pacientes se sometieron a una gastrectomía en manga laparoscópica y otros 18 pacientes se sometieron a un procedimiento robótico utilizando el Da Vinci Surgical System ${ }^{\circledast}$. Se revisaron los datos demográficos de los pacientes, las comorbilidades, la fecha de la cirugía, la morbilidad y mortalidad posoperatorias, el tiempo operatorio y la duración de estancia intrahospitalaria. Resultados: La edad media fue $49.28 \pm 7.62$ (GM-R) frente a $48.83 \pm 6.8$ (GM-L) sin diferencia estadística entre los dos grupos. La demografía de los pacientes fue similar en ambos grupos. El tiempo quirúrgico medio para el grupo robótico fue $79.11 \pm 9.54$ frente a $75.05 \pm 8.67 \mathrm{~min}$ en el grupo laparoscópico, sin diferencia estadística. No hubo diferencias significativas entre los dos

\section{ABSTRACT}

Introduction: Sleeve gastrectomy has gained popularity over the past decade due to its safety, feasibility, and good results. The purpose of this study is to describe our results, short term, with these procedures. Material and methods: This study is a nonrandomized, retrospective review of 36 patients who underwent a minimally invasive sleeve gastrectomy at the National Medical Center 20 de Noviembre from January 2015 to December 2018. A total of 18 patients underwent a laparoscopic sleeve gastrectomy, and another 18 patients underwent a robotic procedure using the da Vinci Surgical System ${ }^{\oplus}$. Patient demographics, comorbidities, date of surgery, postoperative morbidity and mortality, operating time, and length of stay were reviewed. Results: The mean age was $49.28 \pm 7.62$ (R-SL) versus $48.83 \pm 6.8$ (L-SG) with no statistical difference between the two groups. Patient's demographics were similar in both groups. The mean operative time for the robotic group was $79.11 \pm 9.54$ versus $75.05 \pm 8.67$ min in the laparoscopic group, with no statistical difference. There were no significant differences between the two groups with regard to the perioperative complications, length of stay. Conclusion: There is no significant difference between the robotic

Recibido: 14/09/2020. Aceptado: 03/03/2021.

Correspondencia: Dr. Omar Felipe Gaytán Fuentes

Hospital Ángeles Acoxpa, Consultorio 285, Calzada Acoxpa Núm. 430, Col. Ex Hacienda Coapa, 14370, Alcaldía Tlalpan, Ciudad de México, México. E-mail: clinic.obedi@gmail.com

Citar como: Gaytán FOF, Gaytán FIA, Ayala VGA, Barajas GE, Guerrero CJ, Almazán HAl et al. Gastrectomía en manga robótica y laparoscópica: Resultados tempranos de un estudio en población mexicana. Rev Mex Cir Endoscop. 2020; 21 (3): 139-144. https://dx.doi.org/10.35366/99837 
grupos con respecto a las complicaciones perioperatorias y la duración de la estancia. Conclusión: No hay diferencias significativas entre el grupo robótico y laparoscópico en términos de complicaciones, duración de la estadía y pérdida de sangre estimada. La gastrectomía en manga asistida por robot se asocia con mayor tiempo quirúrgico y costo más elevado.

Palabras clave: Gastrectomía en manga asistida por robot, gastrectomía en manga laparoscópica.

\section{INTRODUCCIÓN}

La obesidad es un problema de salud cada vez mayor en las sociedades modernas y la cirugía se ha establecido como el medio más eficaz de tratamiento para pacientes con obesidad mórbida. La cirugía bariátrica y metabólica ofrece pérdida de peso a largo plazo, mejoría de las comorbilidades asociadas como diabetes mellitus, hipertensión arterial, dislipidemia, y una disminución en la mortalidad a largo plazo en los pacientes afectados. ${ }^{1}$ Según las estimaciones más recientes de la Sociedad Americana de Cirugía Metabólica y Bariátrica (ASMBS por sus siglas en inglés), la gastrectomía en manga (GM) es hoy en día la cirugía bariátrica más comúnmente realizada en el mundo, gastrectomía en manga (GM, 45.9\%), derivación gástrica Roux-en-Y (BPG-YR, 39.6\%), banda gástrica ajustable laparoscópica (BGA-L, 7.4\%) y derivación biliopancreática/ switch duodenal (DBP/SD, 1.1\%). ${ }^{2,3}$ La cirugía robótica se ha desarrollado para ampliar las indicaciones de la cirugía de mínimo acceso. Desde principios de la década de 2000, se ha informado que la cirugía robótica es factible y segura, incluso para procedimientos avanzados y complejos. ${ }^{4,5}$ En 2003, Intuitive Surgical (Sunnyvale, CA) se fusionó con Computer Motion (Goleta, CA) creando de manera efectiva un único proveedor robótico. Desde entonces, ha habido avances tecnológicos sustanciales en la plataforma quirúrgica Da Vinci ${ }^{\circledR}$ destinada principalmente a mejorar la eficiencia operativa. ${ }^{6}$ La creciente popularidad de la plataforma robótica Da Vinci ${ }^{\circledR}$ (Intuitive Surgical, Atlanta, GA; EUA) en otras especialidades quirúrgicas ha impulsado su uso en la cirugía bariátrica. ${ }^{7}$ Ésta inicialmente se utilizó para realizar la derivación gástrica y las derivaciones biliopancreáticas, pero ha habido un incremento en el uso de este abordaje para llevar a cabo la manga gástrica. ${ }^{4}$ Actualmente representa $7 \%$ de todas las GM realizadas en la base de datos del Programa de Acreditación y Mejora de la Calidad de la Cirugía Metabólica y Bariátrica (MBSAQIP por sus siglas en inglés). ${ }^{7}$ Uno de los principales argumentos en contra del uso de la plataforma Da Vinci en cirugía bariátrica es que la tecnología tiene un costo inaccesible. ${ }^{8}$ En la literatura actual, las complicaciones relacionadas con and laparoscopic group in terms of complications, length of stay, and estimated blood loss. Robot-assisted sleeve gastrectomy is associated with longer operative time and increased cost.

Keywords: Robot-assisted sleeve gastrectomy, laparoscopic sleeve gastrectomy. los procedimientos bariátricos laparoscópicos se sitúan en promedio de 5 a $10 \%$, siendo la fuga de anastomosis la que mayor porcentaje podría tener en $5.1 \% .{ }^{9}$ Estos porcentajes podrían disminuir con el uso del abordaje robótico, siendo partes sus ventajas las cuales incluyen no sólo el tiempo de recuperación, disminución del dolor y corta estancia hospitalaria, sino también las mejoras para el cirujano, mayor destreza y precisión en la manipulación de los tejidos en sitios de difícil acceso, dando como resultado menor riesgo de conversión de las cirugías. ${ }^{10}$ Haciendo una revisión de la literatura y de los estudios comparativos, aún hay ausencia de ventajas clínicas y presencia de mayor tiempo transoperatorio en la manga gástrica robótica en comparación con el abordaje laparoscópico. ${ }^{11-13}$

Recientemente se ha observado que pacientes con un índice de masa corporal (IMC), $\geq 50 \mathrm{~kg} / \mathrm{m}^{2}$ (superobesos, (SO)) presentan desafíos particulares relacionados tanto con la cirugía como con la anestesia debido a las comorbilidades y características corporales, es así que los procedimientos bariátricos laparoscópicos son en general seguros y efectivos en estos pacientes, pero están asociados con mayor morbilidad y mortalidad a 30 días. ${ }^{14}$ La cirugía laparoscópica en pacientes con SO es técnicamente más difícil como resultado del aumento del grosor de la pared abdominal, el torque excesivo del instrumento, la gran cantidad de grasa periférica y el espacio de trabajo limitado. Los enfoques asistidos por robot tienen el potencial para aliviar varios de estos desafíos y, por lo tanto, son particularmente útiles en casos en los que la laparoscopía tiene deficiencias. ${ }^{15}$

El objetivo de este estudio es informar nuestra experiencia de pacientes sometidos a gastrectomía en manga de mínimo acceso (laparoscópica y robótica) y tratar de identificar cualquier ventaja y desventaja de la técnica que pueda afectar el tratamiento inmediato.

\section{MATERIAL Y MÉTODOS}

Se realizó un estudio retrospectivo comparativo en pacientes con obesidad mórbida que fueron sometidos a gastrectomía en manga en el periodo comprendido de enero de 2015 a febrero de 2019; gastrectomía en manga 
robótica ( $n=18)$, laparoscópica $(n=18)$ como primer procedimiento realizado en la Clínica de Obesidad y Enfermedades Metabólicas del Centro Médico Nacional 20 de Noviembre ISSSTE. Las variables a estudiar en ambos grupos fueron las características demográficas (edad, sexo, peso, talla, índice de masa corporal), perioperatorias (tiempo quirúrgico, estancia hospitalaria, necesidad de terapia intensiva, conversiones y reoperaciones), complicaciones (tempranas y tardías).

Los criterios de inclusión utilizados fueron IMC $<35$ $\mathrm{kg} / \mathrm{m}^{2}$ con la presencia de comorbilidades, edad mayor de 18 años y menor de 65. Los criterios de exclusión fueron pacientes con IMC > $60 \mathrm{~kg} / \mathrm{m}^{2}$, sometidos a cualquier procedimiento bariátrico previo, imposibilidad para completar 30 días de seguimiento o expediente/laboratorios incompletos. Todos los pacientes fueron evaluados por un equipo multidisciplinario, cirujanos, endocrinólogos, nutriólogos y psicólogos para una buena elección de candidatos al procedimiento quirúrgico.

Los resultados fueron expresados en media y desviación estándar (DE), se utilizó estadística descriptiva para las variables demográficas, las diferencias entre los grupos se analizaron mediante la prueba $U$ de Mann-Whitney para las variables cuantitativas. Para la comparación de las características perioperatorias se empleó U de Mann-Whitney. Se consideró una p significativa cuando ésta fue menor a 0.05.

\section{Técnica quirúrgica}

La técnica quirúrgica fue estandarizada y realizada por un mismo cirujano del servicio en ambos grupos, se calibró el estómago con una sonda de 32-40 Fr (media de 36Fr), la antrectomía se inició de 2-6 cm del píloro, terminando a 1-2 cm del ángulo de His, mecánica en su totalidad, terminando con un volumen estimado de 75 a $150 \mathrm{~mL}$, se hizo prueba de hermeticidad con azul de metileno, no se realizó sobresutura de la línea de grapeo.

\section{Implicaciones éticas}

Todos los procedimientos efectuados en este estudio incluyeron participantes humanos, los cuales estuvieron de acuerdo con los estándares éticos del comité de investigación institucional y/o nacional y con la declaración de Helsinki de 1964 y sus enmiendas posteriores o estándares éticos comparables. Para este estudio retrospectivo no se requirió consentimiento formal.

\section{RESULTADOS}

En un periodo de estudio se incluyeron 36 pacientes que cumplían con los criterios mencionados. Durante el seguimiento y análisis a 30 días se obtuvo un porcentaje de seguimiento de $100 \%$ en ambos grupos. Sólo se analizaron los pacientes que completaron el análisis.

\section{Características demográficas}

Las características demográficas basales fueron homogéneas en ambos grupos (Tabla 1). De la muestra, $77.7 \%$ fueron pacientes del sexo femenino en el grupo de GM-R, y $83.3 \%$ en el grupo de GM-L, con una media de edad de $49.28 \pm 7.62$ versus $48.83 \pm 6.8$ años respectivamente $(p=0.855)$. La media de peso e IMC en el grupo de pacientes de GM-R fue de $126.33 \pm 19.79 \mathrm{~kg}$ y $47.64 \pm$ $1.49 \mathrm{~kg} / \mathrm{m}^{2}$ versus $122.38 \pm 19.91 \mathrm{~kg}$ y $46.61 \pm 1.42 \mathrm{~kg} /$

\begin{tabular}{|c|c|c|c|}
\hline & GM-R $(n=18)$ & GM-L $(n=18)$ & $p$ \\
\hline Edad (años) & $49.28 \pm 7.62$ & $48.83 \pm 6.8$ & 0.855 \\
\hline \multicolumn{4}{|l|}{ Sexo } \\
\hline Femenino & 14 & 15 & NA \\
\hline Masculino & 4 & 3 & \\
\hline Peso (kg) & $47.64 \pm 1.49$ & $46.61 \pm 1.42$ & 0.554 \\
\hline IMC & $126.33 \pm 19.79$ & $122.38 \pm 19.91$ & 0.041 \\
\hline Hipertensión, n (\%) & 4 (22.2) & 4 (22.2) & 1.000 \\
\hline DM2, n (\%) & $6(33.3)$ & $4(22.2)$ & 0.471 \\
\hline $\mathrm{TG}>150 \mathrm{mg} / \mathrm{dL}, \mathrm{n}(\%)$ & $9(50.0)$ & $11(61.1)$ & 0.516 \\
\hline $\mathrm{CT}>200 \mathrm{mg} / \mathrm{dL}, \mathrm{n}(\%)$ & $5(27.7)$ & $4(22.2)$ & 0.710 \\
\hline $\mathrm{HDL}<40 \mathrm{mg} /<50 \mathrm{mg}, \mathrm{n}(\%)$ & $8(44.4)$ & $10(50.0)$ & 0.519 \\
\hline
\end{tabular}

Características basales de los pacientes, los valores muestran la media \pm desviación estándar para las características preoperatorias y el porcentaje. $\mathrm{GM}-\mathrm{R}$ = gastrectomía vertical en manga robótica, $\mathrm{GM}-\mathrm{L}$ = gastrectomía vertical en manga laparoscópica, IMC = índice de masa corporal; DM2 = diabetes mellitus tipo 2, TG = triglicéridos, $\mathrm{HDL}=$ colesterol de alta densidad, $\mathrm{CT}=$ colesterol total. 
Tabla 2: Características perioperatorias y complicaciones de ambos grupos.

\begin{tabular}{lccc} 
& GM-R $(n=18)$ & GM-L $(n=18)$ & $p$ \\
\hline Características perioperatorias & & & \\
$\quad$ Tiempo quirúrgico & $79.11 \pm 9.54$ & $3.3 \pm 0.485$ & 0.179 \\
Estancia hospitalaria & $75.05 \pm 8.67$ & $3.5 \pm 0.514$ & 0.324 \\
Conversión & 0 & 0 & \\
UCl & 0 & 0 & 0.145 \\
Reintervención & 0 & $4(4.2)$ & \\
Total de complicaciones & $3(3.1)$ & & \\
$\quad$ Complicaciones menores $n(\%)$ & &
\end{tabular}

$\mathrm{m}^{2}$ en el grupo de pacientes con GM-L respectivamente $(\mathrm{p}$ $=0.554, \mathrm{p}=0.041$.

\section{Características perioperatorias}

El tiempo quirúrgico y estancia hospitalaria en ambos grupos fue similar, en el grupo de GM-R con una media 79.11 \pm 9.54 minutos y $3.3 \pm 0.485$ días respectivamente, en el grupo de GM-L con una media de $75.05 \pm 8.67$ minutos y $3.5 \pm 0.514$ días respectivamente $(p=0.719, p=0.324)$. No hubo ningún paciente que requiriera estancia en terapia intensiva ni conversión a cirugía abierta en ambos grupos (Tabla 2).

\section{Complicaciones menores de $\mathbf{3 0}$ días}

Un total de 10 pacientes (27.7\%) tuvieron una complicación menor, tres pacientes (16.6\%) en el grupo de GM-R y siete $(38.8 \%)$ en el grupo de GM-L ( $p=0.265)$, en ambos grupos fue la presencia de dolor abdominal. No hubo complicaciones mayores tempranas.

\section{DISCUSIÓN}

El abordaje laparoscópico para la manga gástrica sigue siendo el estándar de oro para este procedimiento, dejando al abordaje robótico como una alternativa usada en centros especializados. ${ }^{16}$ Aunque cada vez es más utilizado, el uso de la plataforma robótica en la cirugía bariátrica es controvertido, y no hay evidencia de nivel I que sugiera si es mejor o peor en comparación con la laparoscopía convencional, algunos investigadores informaron mejores resultados y otros informaron resultados similares o peores. ${ }^{8}$

El abordaje laparoscópico tiene una serie de desventajas que incluyen visión bidimensional, falta de destreza debido al diseño rígido del instrumento, movimiento de rotación limitado, profundidad de visión reducida y ergonomía desafiante. ${ }^{17}$ Los defectos del abordaje de laparoscopía en pacientes con obesidad grado III o superobesidad conducen a un procedimiento técnicamente más desafiante como resultado de la pared abdominal más gruesa, la mayor cantidad de grasa visceral en la cavidad abdominal y el hígado graso que disminuyen el espacio de trabajo. ${ }^{14}$ Las ventajas de la cirugía robótica sobre la laparoscopía tradicional incluyen imágenes tridimensionales, escala de movimiento y filtración de temblores, mejorando así la destreza y precisión, siete grados de libertad y una ergonomía mejorada. ${ }^{18,14}$

En la mayoría de otras comparaciones entre el abordaje robótico y laparoscópico se ha observado un aumento del tiempo operatorio con el enfoque robótico, incluido un pequeño estudio centrado en pacientes superobesos. El aumento en el tiempo operatorio está relacionado con el atraque y el desacoplamiento, además de ser altamente dependiente de la experiencia del cirujano y del personal operativo. ${ }^{13}$ Nuestros resultados siguen esa tendencia, mostrando un aumento de tiempo en el abordaje robótico versus el laparoscópico $(79.11 \pm 9.54$ minutos en el grupo GM-R versus $75.05 \pm 8.67$ minutos EN GM-L).

Respecto a la estancia hospitalaria nuestros datos son similares a los reportados por Romero y colaboradores, ${ }^{12}$ teniendo para el grupo de GM-R una estancia hospitalaria significativamente más corta que los pacientes que se sometieron a GM-L (2.2 días versus 3.3 días), en nuestra institución se registró 3.3 días versus 3.5 días respectivamente. Hay estudios que demuestran estancia hospitalaria más corta como lo describen Magouliotis y su equipo ${ }^{19} \mathrm{con}$ un media en el grupo de GM-R de 2.8 días versus 3.55 días en GM-L. Asimismo, se ha reportado que no existe diferencia entre el tiempo de estancia hospitalaria como lo describen Elli y colegas. ${ }^{15}$

En nuestra población no hubo reingresos hospitalarios dentro de los primeros 30 días ni reoperación o presencia de fugas, similar a lo descrito por Moon y colaboradores, ${ }^{20}$ 
quienes evaluaron los resultados de su centro antes y después de un periodo de curva de aprendizaje y no encontraron diferencias en el reingreso, tasa de reoperación y fugas en los primeros 30 días.

El abordaje robótico no fue superior al laparoscópico, y no debe causar sorpresa, la duración no es inferior al tiempo laparoscópico, en cuanto a estancia hospitalaria y mortalidad tiene resultados similares, lo que debe interesarnos son las tendencias hacia tasas más bajas, aunque no significativas, de complicaciones y reoperaciones dentro de los primeros 30 días, ya que pueden reflejar posibles beneficios reales en el abordaje laparoscópico dada la mayor experiencia del cirujano y el desarrollo tecnológico.

El estudio actual no pudo comparar los costos de GM-R versus GM-L, ya que los datos de costos no están disponibles en la base de datos de nuestra institución. Los estudios de Moon y su equipo ${ }^{20}$ y Vilallonga y colegas ${ }^{11}$ demuestran costos más altos para la GM-R (rango de \$10,556 a \$ 56,464 dólares) en comparación con GM-L (rango de \$ 8795 a \$ 49,498 dólares).

Actualmente, no hay un número establecido que dé cuenta de la competencia en la gastrectomía en manga robótica, y la base de datos MBSAQIP no proporciona datos sobre la experiencia del cirujano en este abordaje, llegando a la conclusión de que la cirugía robótica se puede realizar de manera segura en hospitales especializados y en el marco de programas de capacitación para residentes sin un impacto negativo en el resultado. ${ }^{3}$

Nuestro estudio es uno de los pocos reportes comparativos sobre la gastrectomía vertical en manga robótica versus laparoscópica en el país. A pesar de contar con la tecnología del sistema Si y Xi de la plataforma Intuitive Surgical Da Vinci, en nuestro medio no existen estándares de calidad sobre su uso, por lo cual nuestro análisis tiene importantes limitaciones. Primero, este estudio es de un solo centro que utiliza datos específicos de la institución con un seguimiento breve (sólo 30 días), por lo que nuestras conclusiones no pueden generalizarse, ya que las técnicas quirúrgicas son personalizadas en cada centro. Además, aunque nuestra muestra es comparable, los grupos no se pueden estandarizar debido al bajo número de participantes en cada uno. Continuaremos recopilando nuestros datos e informaremos los resultados a largo plazo (más de 30 días). En segundo lugar, los resultados se basaron utilizando una antigua plataforma de $\mathrm{Si}$, desde entonces, se han actualizado nuevas plataformas (Xi) que tienen ventajas técnicas que pueden resultar incluso en más tiempo y ahorro de costos. Tercero, el tamaño pequeño de nuestra muestra puede haber llevado a un error tipo II, es posible que pacientes adicionales produzcan una diferencia significativa en los tiempos y complicaciones posoperatorias entre GM-R y GM-L.

\section{CONCLUSIÓN}

Aunque al inicio había grandes esperanzas de mejoras teóricas de la cirugía robótica en las técnicas laparoscópicas, el impacto en la calidad y los resultados de la cirugía bariátrica a menudo han estado por debajo de las expectativas. Si bien algunas instituciones han encontrado formas rentables de utilizar la plataforma robótica para mejorar sus resultados, todavía no han surgido hallazgos unánimes que muestren indicaciones claras de que la cirugía robótica supera a la laparoscopía en bariatría. Es probable que esto se deba a que la laparoscopía ya era adecuada para realizar procedimientos de pérdida de peso con una tasa de complicaciones ciertamente baja. Parece que los casos laparoscópicos difíciles son casos robóticos igualmente difíciles y los datos han mostrado poca mejora en el tiempo o los costos. De hecho, en casi todos los estudios se encontró que los costos eran sustancialmente más altos cuando se utilizó el robot.

La gastrectomía en manga asistida por robot representa un porcentaje pequeño, pero creciente del número total de procedimientos realizados en nuestro país. A medida que nos adaptemos a las nuevas tecnologías, es recomendable implementar el monitoreo continuo y la presentación de informes con datos de seguridad y resultados para mantener y reportar los altos estándares de resultados en cirugía bariátrica.

\section{REFERENCIAS}

1. Andreas A, Adamantios M, Antonios A, Theofilos R, Christos T, Theodoros D. Laparoscopic sleeve gastrectomy for morbid obesity with intra-operative endoscopy: Lessons we learned after 100 consecutive patients. Obes Surg. 2015; 25: 1223-1228. doi: 10.1007/s 1 1695-014-1524-3.

2. Angrisani L, Santonicola A, lovino P et al. Bariatric surgery and endoluminal procedures: IFSO worldwide survey 2014. Obes Surg. 2017; 27: 1-11. doi: 10.1007/s ${ }^{1} 1695-$ 017-2666-x.

3. Bhandari M, Fobi MAL, Buchwald JN et al. Standardization of bariatric metabolic procedures: World consensus meeting statement. Obes Surg. 2019; 29: 309-345. doi: 10.1007/ $\mathrm{s}^{1}$ 1695-019-04032-x.

4. Buchs NC, Morel P, Azagury DE et al. Laparoscopic versus robotic roux-en-y gastric bypass: Lessons and long-term follow-up learned from a large prospective monocentric study. Obes Surg. 2014; 24: 2031-2039. doi: 10.1007/ s'1695-014-1335-6.

5. Buchs NC, Addeo P, Bianco FM et al. Perioperative risk assessment, in robotic general surgery: Lessons learned from 884 cases at a single institution. Arch Surg. 2012; 147: 701-708. doi: 10.1001/archsurg.2012.496.

6. Dudash M, Kuhn J, Dove J et al. The longitudinal efficiency of robotic surgery: an MBSAQIP propensity matched 4-year 
comparison of robotic and laparoscopic bariatric surgery. Obes Surg. 2020; 30: 3706-3713. doi: 10.1007/s $1695-$ 020-04712-z.

7. Lundberg PW, Stoltzfus J, El Chaar M. 30-day outcomes of robot-assisted versus conventional laparoscopic sleeve gastrectomy: First analysis based on MBSAQIP. Surg Obes Relat Dis. 2019; 15: 1-7. doi: 10.1016/j.soard.2018.10.015.

8. El Chaar M, Gacke J, Ringold S, Stoltzfus J. Cost analysis of robotic sleeve gastrectomy (R-SG) compared with laparoscopic sleeve gastrectomy (L-SG) in a single academic center: debunking a myth! Surg Obes Relat Dis. 2019;15: 675-679. doi: 10.1016/j.soard.2019.02.012.

9. DeMaria EJ, Sugerman HJ, Kellum JM, Meador JG, Wolfe LG. Results of 281 consecutive total laparoscopic Rouxen-Y gastric bypasses to treat morbid obesity. Ann Surg. 2002; 235: 640-647. doi: 10.1097/00000658-20020500000005.

10. Jung MK, Hagen ME, Buchs NC, Buehler LH, Morel P. Robotic bariatric surgery: A general review of the current status. Int J Med Robot Comput Assist Surg. 2017; 13: 1-8. doi: 10.1002/rcs.1834.

11. Vilallonga R, Fort JM, Caubet E, Gonzalez O, Armengol M. Robotic sleeve gastrectomy versus laparoscopic sleeve gastrectomy: A comparative study with 200 patients. Obes Surg. 2013; 23: 1501-1507. doi: 10.1007/s¹695-0131039-3.

12. Romero RJ, Kosanovic R, Rabaza JR et al. Robotic sleeve gastrectomy: Experience of 134 cases and comparison with a systematic review of the laparoscopic approach. Obes Surg. 2013; 23: 1743-1752. doi: 10.1007/s $1695-013-$ 1004-1
13. Ayloo S, Buchs NC, Addeo P, Bianco FM, Giulianotti PC. Robot-assisted sleeve gastrectomy for super-morbidly obese patients. J Laparoendosc Adv Surg Tech. 2011; 21: 295-299. doi: 10.1089/lap.2010.0398.

14. Nasser H, Ivanics T, Ranjal RS, Leonard-Murali S, Genaw J. Perioperative outcomes of robotic versus laparoscopic sleeve gastrectomy in the superobese. J Surg Res. 2020; 249: 34-41. doi: 10.1016/j.jss.2019.12.012

15. Elli E, Gonzalez-Heredia R, Sarvepalli S, Masrur M. Laparoscopic and robotic sleeve gastrectomy: short- and long-term results. Obes Surg. 2015; 25: 967-974. doi: 10.1007/s ${ }^{1} 1695-014-1499-0$.

16. Bindal V, Bhatia P, Dudeja. Review of contemporary role of robotics in bariatric surgery. J Minim Access Surg. 2015; 11 : 16-21. doi: 10.4103/0972-9941.147673.

17. Lee S, Carmody B, Wolfe L. Effect of location and speed of diagnosis on anastomotic leak outcomes in 3828 gastric bypass cases. J Gastrointest Surg. 2007; 11: 708-713. doi:10.1007/s ${ }^{1} 1605-007-0085-3$.

18. Choussein S, Srouji SS, Farland LV. Robotic Assistance Confers Ambidexterity to Laparoscopic Surgeons. J Minim Invasive Gynecol. 2018; 25: 76-83. doi: 10.1016/j. jmig.2017.07.010.

19. Magouliotis DE, Tasiopoulou VS, Sioka E, Zacharoulis D. Robotic versus Laparoscopic Sleeve Gastrectomy for Morbid Obesity: a Systematic Review and Meta-analysis. Obes Surg. 2017; 27: 245-253. doi: 10.1007/s ${ }^{1} 1695-016-2444-1$.

20. Moon RC, Stephenson D, Royall NA, Teixeira AF, Jawad MA. Robot-assisted versus laparoscopic sleeve gastrectomy: learning curve, perioperative, and short-term outcomes. Obes Surg. 2016; 26: 2463-2468. doi: 10.1007/s¹695-016-2131-2. 